\title{
Data-centric communication strategy for wireless sensor networks
}

\section{Tetsuro Kuniyasu}

Program in Information and Management Systems,

Graduate School of Comprehensive Scientific Research,

Prefectural University of Hiroshima, Japan

Email: q622006vf@ed.pu-hiroshima.ac.jp

\section{Tetsuya Shigeyasu*}

Department of Management Information Systems,

Prefectural University of Hiroshima, Japan

Email: sigeyasu@pu-hiroshima.ac.jp

*Corresponding author

\begin{abstract}
Recently, the popularity of the internet of things technology has highlighted the importance of performance for gathering data over multi-hop wireless sensor nodes. Data obtained from the sensor nodes is used by individual as well as multiple users. Therefore, a data-centric communication strategy is ideal for efficient data gathering and delivery. This paper proposes the implementation of a new data-centric communication strategy based on the named data network (NDN) to wireless sensor network (WSN) for achieving efficient communication performance. We discuss the modification of the NDN, which is originally developed for a wired network, for achieving better communication performance even in wireless communication networks. Specifically, we discuss the methods for constructing the reverse path of the required contents and for selecting the adequate next hop relay node of the forwarding interest. We propose new methods considering the broadcast nature of the wireless channels. Using computer simulations, we confirm that our proposed methods reduce the redundant transmissions by selecting a proper route based on network conditions.
\end{abstract}

Keywords: data-centric communication; data collecting system; in-network cache; wireless sensor network; WSN.

Reference to this paper should be made as follows: Kuniyasu, T. and Shigeyasu, T. (2018) 'Data-centric communication strategy for wireless sensor networks', Int. J. Space-Based and Situated Computing, Vol. 8, No. 1, pp.30-39.

Biographical notes: Tetsuro Kuniyasu received his Bachelor degree from the Prefectural University of Hiroshima in 2016. He is currently a graduate student at the graduate school of Prefectural University of Hiroshima. His research interests include wireless communication systems under ad-hoc networks.

Tetsuya Shigeyasu received his $\mathrm{PhD}$ in Engineering from the Osaka University in 2010 . He became an Associate Professor at the Prefectural University of Hiroshima. His current research interests include wireless communication protocols under distributed wireless networks. He received the Best Paper Award and the Outstanding Oral Paper Award from the DPSWS2007 and ITC-CSCC2008, respectively. He was the Chief Examiner of the IPSJ journal in 2016, and is a member of IEEE and IEICE, and a senior member of IPSJ.

This paper is a revised and expanded version of a paper entitled 'Data collecting system based on CCN with congestion avoidance routing on WSN' presented at The 20th International Conference on Network Based on Information System (NBiS-2017), Toronto, Canada, 24-26 August 2017.

\section{Introduction}

Information exchange over a traditional communication network requires specifying the destination node address, which is allocated according to the location of the nodes.
Hence, this type of communication routing is called location-centric networking. In a traditional network, users want to communicate with each other for remote controlling. However, recent communication networks are used for content delivery in most cases. Here, network users 
obtain their desired contents, such as video, image, and other contents, which are useful for multiple users, i.e., versatile data.

For multiple users, the locations of the nodes returning the content are not important. Most users are only interested in the content of the obtained data in the current network. Therefore, this type of network is called content-centric networking (CCN) (Yamamoto, 2014).

$\mathrm{CCN}$ has been investigated in several studies. The most important concept of $\mathrm{CCN}$ is the change of identification from IP address to content name. By specifying the content name instead of the IP address, users requesting the content can obtain the data from the cache of the closest node. This process significantly reduces the amount of network traffic and response time.

Recently, services related to the internet of things (IoT) (Matsui et al., 2016) have been widely deployed. Services based on the IoT can connect everything to the Internet, which provides cost reduction and effective services to users. Specifically, these services are focused on the development of wireless sensor networks (WSNs) (Tobe, 2007) with a number of sensors. The WSN collects data from remote nodes over multi-hop wireless links without installation of any fixed wired infrastructure. Hence, by building the WSN on the sensing areas, we can establish an effective data collection system at lower cost.

To effectively collect data on a WSN, Intanagonwiwat et al. (2003) proposed the communication protocol called directed diffusion (DD). This protocol broadcasts the information request when a sink wants to obtain data. If the wireless node with a corresponding sensor receives the request, the node delivers the data during a given period over the lowest delay route established by the prior request. However, the DD protocol is required to recognise the neighbouring nodes of each node along the data transfer route. In addition, it induces unnecessary data transfers, which increases the network traffic.

Several studies have reported the implementation of the named data network (NDN) (NDN Project, 2010) on WSN (Amadeo et al., 2013, 2014; Hail et al., 2015; Zhang et al., 2015). Amadeo et al. (2013) reported that the implementation of NDN on WSN ensures data collection over a large-scale WSN using the layered naming of NDN.

However, Amadeo et al. (2013) only described the simple concept of implementing the NDN on WSN, and did not discuss the adequate control sequences for WSN. Therefore, in this paper, we discuss and clarify the two critical issues arising if the original NDN is simply applied to WSN. To mitigate the effect of this problem, this paper proposes new protocols. Specifically, this paper focuses on discussions for adaptive route selection mechanism for the frequent link-changing environment of the wireless network.

To clarify the applicability of the proposed methods, we conducted computer simulations and showed the advantages of the proposed methods.

\section{Related works}

\subsection{Basic procedure of NDN}

In NDN, users obtain the desired contents according to the request/forwarding sequence, which is performed using two packet types: interest and data. First, a user wishing to obtain contents transmits the Interest with the name of the contents. The Interest traverses the network by multi-hop routing. The node that received the Interest checks whether it has the requested content. If the node does not have the requested content, it registers the requested information to the pending interest table (PIT) on the node. The requested information consists of the name of the content and the incoming interface. At this time, if an entry with similar content already exists in the PIT, only the interface number will be added to the existing entry. After the registration, the Interest will be forwarded to the next upstream node. After the interest traversed the network according to the above-mentioned mechanism, the node that has the corresponding data receives the Interest, and sends back the data to the downstream node using the entry on the PIT. During the data transfer to the original node, the intermediate node relaying the data copies it to their content store (CS) if it is available.

If a node receiving the Interest has the corresponding cached data, it will send back its cached data to reduce both network traffic and response time for the user requesting the contents. In addition, to hold the shortest location to the contents, the intermediate nodes add the interface ID of the arriving data into the forwarding information base (FIB). Hence, the Interest can arrive at the node with the desired contents at the shortest route even if the intermediate nodes do not have a copy of the contents.

Figure 1 Example of NDN procedure (see online version for colours)

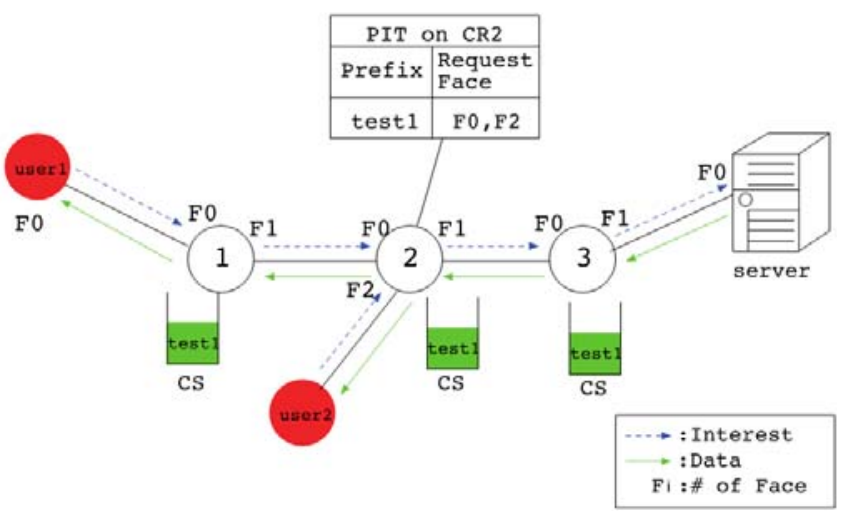

Figure 1 shows an example of the NDN procedure. Let us consider that user1 and user2 request the same content 'test1'. The requests arrive at node 2 in the order of user1 and user2. Node2 forwards the Interest received from user1 to the upstream node. After forwarding the interest of 'test1', node2 registers to its PIT information that 'test1' is 
also requested by user2. When the Interest is received at the original server or the node with the desired cache, the server/node returns the content to the requesting node. At the return path, each node will cache the content for responding to future requests. Thereafter, if 'test1' is requested by another user, it is possible to respond quickly.

\subsection{Application of NDN technology to WSN}

The original NDN technology is intended to be used on the wired network. Therefore, in the previous subsection, we described the basic procedure of NDN on a wired network. However, in our study, we proposed to introduce the NDN to WSN. In this subsection, we define the basic NDN protocol for wireless network as follows:

- The communication method employs CSMA/CA (carrier sense multiple access/collision avoidance) (Editors of IEEE, 1999) of IEEE802.11, which is the most widely used wireless LAN standard.

- The interest will be broadcasted if there is no corresponding entry to the received interest on the receiver FIB.

- Otherwise, the Interest will be transmitted in a unicast manner if there is a corresponding entry to the received interest on the receiver FIB.

- $\quad$ By registering the neighbours' MAC address to PIT and FIB, each node identifies the forwarding destination.

\subsection{Related works proposing the implementation of NDN on wireless networks}

\subsubsection{Kite: a mobility support scheme for NDN}

Kite (Zhang et al., 2014) has been proposed to implement NDN on wireless networks with terminal mobility. Kite aimed for supporting to keep reach abilities under terminal mobility. Specifically, Kite performs packet delivery to the information requester and content holder regardless of the dynamically changing network topology.

For example, when a mobile node $(\mathrm{MN})$ acts as a consumer, in other words, it downloads the contents (download scenario), the MN can obtain the desired content from the neighbouring routers with cache content even if the $\mathrm{MN}$ moves before receiving the content from the producer.

On the other hand, when an $\mathrm{MN}$ acts as a producer (upload/push scenario), it cannot receive an Interest forwarded according to the FIB if it moves away before receiving the interest. Then, Kite offers a mechanism to support in achieving the packet handshake even in this situation.

In Kite, two new types of Interests are introduced. Traced interest is transmitted by $\mathrm{MN}$, which is used for registering the migration path information of the $\mathrm{MN}$ into the relay nodes along the path between $\mathrm{MN}$ and the Corresponding Node $(\mathrm{CN})$. The tracing interest transmitted by $\mathrm{CN}$ traces the path according to the migration path recorded by the traced interest.
In these two interests, three new fields are added as follows:

- TraceName: name of the target Interest for tracing.

- TraceOnly: this flag determines how the interest should be transmitted. The default state of this flag is unset, and the interest will be forwarded according to the information on the FIB and PIT. When the flag is set, the interest will be transmitted according to the PIT information only if the corresponding route exists. Otherwise, the interest will be forwarded according to the FIB.

- Traceable This flag enables the tracing of the path information. If the flag is unset, the trace will not be allowed.

In addition to the new Interest format, two tables are added for the NDN on Kite. The first is a trace forwarding table (TFT), which records the path of the forwarded interest with traceable option. After the construction of TFT, the tracing interest will be forwarded according to this information. The second is the trace name table (TNT), which records the information of the received tracing interest if the Interest could not be forwarded to the next relay node because of the lack of corresponding TFT entry. When a node has an entry on its TNT corresponding to the newly received Traced Interest, it can return the tracing interest towards the direction of the arriving traced interest.

Figure 2 Migration detection of MT on kite

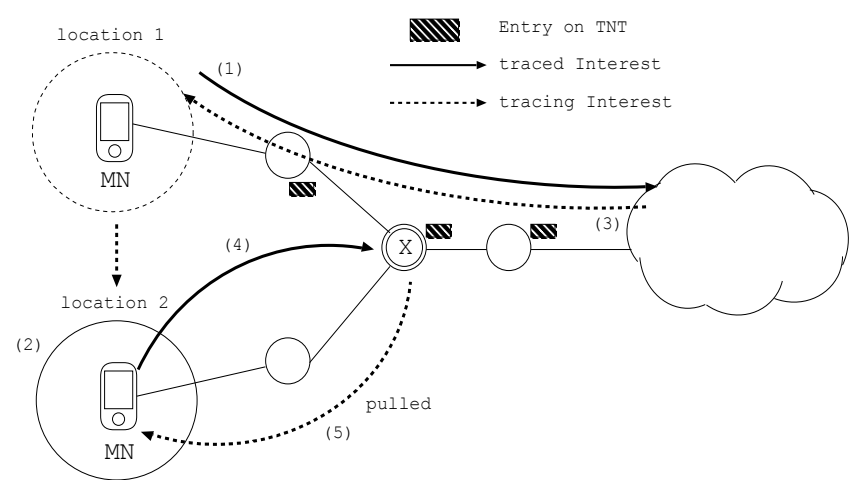

To explain the control sequence of Kite, let us consider the situation in Figure 2 where

\section{$1 \mathrm{MN}$ periodically transmits the traced interest to $\mathrm{CN}$}

2 After the transmission, the route to the $\mathrm{MN}$ is changed by the migration of the MN

3 When the traced interest arrives at the $\mathrm{CN}$, it returns the tracing interest to confirm the location of the MN.

The transmitted traced interest will be forwarded according to the information of the entry stored in the TFT of the relay routers. At this time, if the $\mathrm{MN}$ already moved to a place other than the original location, the tracing interest cannot reach the $\mathrm{MN}$ as indicated by (4) and (5) in the figure. However, the relay nodes hold the forwarded tracing interest in its TNT. Then, if the new traced interest from the 
$\mathrm{MN}$ at the new location will be received by the relay nodes, they can re-forward the past-received traced interest to the MN correctly. This re-forward sequence is called pull. Using the pull sequence, kite can cope with the dynamically changing topology.

\subsubsection{Last encounter routing}

Last encounter routing (LER) is the protocol for introducing the NDN to the vehicular ad-hoc networks (VANET) (Yu et al., 2013). To introduce the NDN to VANET, the network has to cope with the migration of cached contents. To increase the availability of the NDN on VANET, the scalabilities of routing for interest/contents rendezvous must be increased.

In LER, nodes moving around the network exchange their cached contents location list when they contact the other nodes. By updating the contents list continuously, LER improves the accuracy of Interest forwarding.

If the Interest is received by the mobile nodes, the node with the cached content location corresponding to the interest forwards the Interest by geo-routing as shown in Figure 3.

Figure 3 Procedure of LER

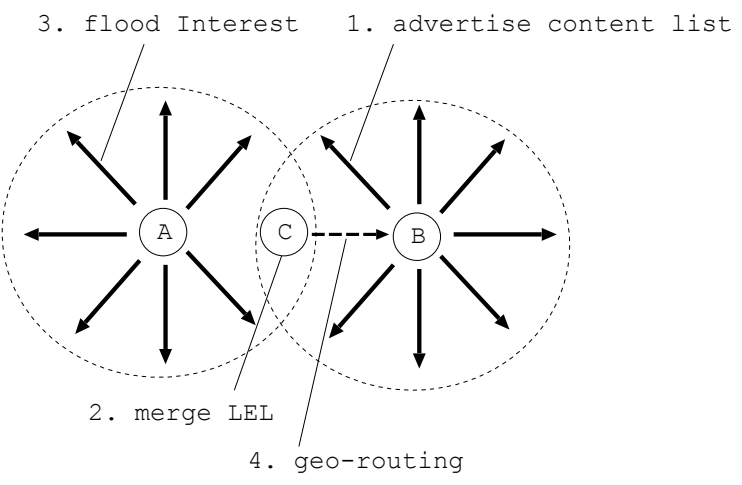

\section{Adaptive relay node selection method on WSN}

Based on Kuniyasu and Shigeyasu (2017), we discussed the implementation of NDN on WSN for coping with the vulnerable wireless nature in which the transmitted packet is frequently lost because of the noisy channel.

\subsection{Dynamic change forwarding in selecting the next relay node according to the next node}

Based on the discussion in Kuniyasu and Shigeyasu (2017), we proposed the new adaptive relay node selection method on WSN called dynamic change forwarding (DCF).

DCF changes the relay node, which forwards the Interest. As the criterion used for the selection, DCF can be categorised into four variants: $\mathrm{DCF}_{\text {ratel }}, \mathrm{DCF}_{\text {rate2 }}, \mathrm{DCF}_{\text {timel }}$, and $\mathrm{DCF}_{\text {time2 }}$. The first two methods, $\mathrm{DCF}_{\text {rate } 1}$ and $\mathrm{DCF}_{\text {rate2 }}$, decide the adequate next relay node according to the return ratio of ACK corresponding to the interest. The latter two methods, DCFtime1 and DCFtime2, decide according to the average length of time required for successful transmission of the Interest.

All four variants of DCF obtain the status information only from its neighbour node.

Four sequences described as followings.

a $\mathrm{DCF}_{\text {ratel }}$ : in this sequence, at every MAC level ACK reception, node calculates the average $\mathrm{ACK}$ reception ratio $\mathrm{R}$ for the last $\mathrm{N}$ ACKs. Here, value of $\mathrm{N}$ is given by the network operator.

b $\mathrm{DCF}_{\text {rate2 }}$ : this sequence selects best route according to MAC level ACK reception ratio $\mathrm{R}$ for last $\mathrm{N}$ ACKs. This sequence, however, calculates the ACK reception rate at every $\mathrm{N}$ ACK receptions.

c DCF $_{\text {timel }}$ : this sequence selects best route according to average MAC level ACK turnaround time for last $\mathrm{N}$ ACKs. The average turnaround time is calculated at every ACK reception.

d $\mathrm{DCF}_{\text {time2 }}$ : this sequence selects best route according to the average MAC level ACK turnaround time for last $\mathrm{N}$ ACKs. This sequence, however, calculates the average turnaround time at every $\mathrm{N}$ ACK receptions.

As the above descriptions, sequences $\mathrm{DCF}_{\text {rate2 }}$ and $\mathrm{DCF}_{\text {time2 }}$ judge the better route at longer intervals than the $\mathrm{DCF}_{\text {ratel }}$ and $\mathrm{DCF}_{\text {timel }}$.

Based on the computer simulations, we have clarified that $\mathrm{DCF}_{\text {rate1 }}$ and $\mathrm{DCF}_{\text {rate2 }}$ can have higher performance than the simple NDN method without the adaptive relay node selection method and the other two types of DCF. In addition, we have concluded that $\mathrm{DCF}_{\text {rate } 2}$ is a more suitable method than DCFrate1 because it can achieve an equally high performance with $\mathrm{DCF}_{\text {rate } 1}$ with lesser number of wireless link investigations.

However, these four methods were evaluated under the condition where the number of relay nodes is not changed. Therefore, the characteristics of the methods obtained by previous studies are limited.

\subsection{DCF with path level investigation}

As discussed in the previous section, the four variants of DCF proposed in Kuniyasu and Shigeyasu (2017) only considered the status information of its neighbour nodes. Therefore, it might not provide the adequate decision to select the forwarding route when the candidate route consists of multiple relay nodes. This means that the four variants of DCF cannot perceive that the candidate route is not adequate even if the relay node is exposed by the interference.

Therefore, in this paper, we proposed two new variants of DCF that consider the status information of their neighbours at least two hops apart from the selector node. 


\subsection{1 $D C F_{2 h o p}$}

First, we proposed a method called $\mathrm{DCF}_{2 \mathrm{hop}}$, which considers the status information of the relay node two hops a part from the selector node. To select the forwarding route from the candidates, $\mathrm{DCF}_{2 \text { hop }}$ proceeds with the following steps:

1 A node that has multiple forwarding routes of Interest becomes the multi-path CR (MPCR).

2 The neighbouring relay nodes to the MPCR (CRneighbour) calculate the ACK reception ratio $R$ corresponding to its interest.

3 CRneighbour adds the value of $R$ to the data returned to the MPCR.

4 The MPCR can obtain the status information of the wireless link of CRneighbour by checking the data returned from the CRneighbour. Then, the MPCR can select the appropriate next relay node based on this information.

\subsubsection{DCF path}

Next, we proposed a method called $\mathrm{DCF}_{\text {path }}$, which considers the status information of all relay nodes belonging to the candidate path.

In the $\mathrm{DCF}_{\text {path }}$, the check packet $(\mathrm{CP})$ is used for checking the status information of the entire candidate path. The CP has the same frame format with the data without the payload. However, it adds the check flag with 1-bit Boolean flag. When the CP is transmitted to the server with the original content of the corresponding content name, all relay nodes are only used for carrying the value of $\alpha$.

The procedures of the DCFpath are as follows.

1 A user transmits CP to the selected route at a specific period.

2 A CR receiving a CP from its downstream CR calculates the $R$. If the $\mathrm{CR}$ is the topmost $\mathrm{CR}$, the $\mathrm{CP}$ is simply discarded.

3 If the $R$ calculated in the previous step is larger than the $\alpha$ recorded in the $\mathrm{CP}$, the $\mathrm{CR}$ registers the information on the PIT, and the CR forwards the CP to the next upstream CR. Otherwise, the CR returns the $\mathrm{CP}$ to the downstream $\mathrm{CR}$ towards where the user originally transmitted the CP.

4 The MPCR receives the returned $\mathrm{CP}$, and changes the forwarding Interest route to another route selected from the candidate routes.

\subsection{DCF for cache-centric forwarding}

In the previous section, we proposed the DCF variants, called $\mathrm{DCF}_{\text {path, }}$, for selecting a suitable forwarding destination by investigating the entire path of candidate routes.
However, the $\mathrm{DCF}_{\text {path }}$ only considers the channel status of the nodes consisting the candidate routes. Hence, it could not select the better candidate route even if a candidate route with caches of popular contents frequently requested by the consumers exists. Therefore, in this section, we proposed another variant of $\mathrm{DCF}$, called $\mathrm{DCF}_{\text {loss, }}$, which decides the forwarding destination node according to the conditions of what contents are found in the relay nodes.

$\mathrm{DCF}_{\text {loss }}$ investigates the Interest inflow rate of each relay node consisting the candidate routes in addition to the Interest loss rate at the $\mathrm{DCF}_{\text {path }}$. Using these two factors on the relay nodes, the $\mathrm{DCF}_{\text {loss }}$ can select the better route compared with the $\mathrm{DCF}_{\text {path }}$ with higher accuracy. Specifically, $\mathrm{DCF}_{\text {loss }}$ can select the route with more caches of popular contents even if the route contains any relay node with possibility of packet loss.

Figure 4 Path status investigation on $\mathrm{DCF}_{\text {loss }}$ (see online version for colours)

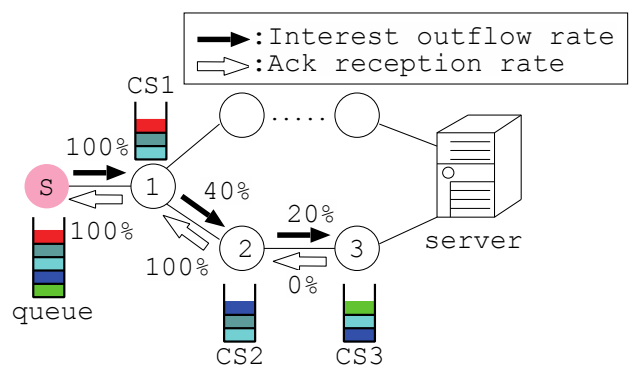

Figure 4 shows the process of the path status investigation on the $\mathrm{DCF}_{\text {loss. }}$. The values on the black arrows show the interest outflow rate $F$, and the values under the white arrows show the ACK reception rate $R$. $F$ denotes the ratio of the arrived interest generated at the consumer $S . R$ denotes the ACK reception rate for checking the packet loss status of the relay node similar to the $\mathrm{DCF}_{\text {path }}$. Both $F$ and $R$ are calculated at each relay node. For each investigation period $T$, the consumer sends the $\mathrm{CP}$ towards the server. At this time, the number of generated interest by the consumer is recorded in this $\mathrm{CP}$ for calculating $F$ at each relay node receiving the $\mathrm{CP}$.

The relay node receives the $\mathrm{CP}$, derives the interest loss rate $L$ according to $F$ and $R$ using equation (1).

$$
\mathrm{L}=(1-\mathrm{R}) \times \mathrm{F}
$$

If the derived $L$ becomes smaller than $\beta$, which is given by the network operator, the relay node returns the CP to the consumer. The MPCR confirms that the interface that received the returned $\mathrm{CP}$ is not the suitable route for Interest forwarding.

Equation (1) shows that the value of $\mathrm{L}$ is influenced by $F$. The values of the $F$ relay nodes close to the server will be small when the relay nodes close to the consumer have many caches of popular contents.

Hence, our proposed $\mathrm{DCF}_{\text {loss }}$ can select the route and improve the cache hit performance even if the relay nodes close to the server are in bad channel condition. 


\section{Performance evaluation}

In this section, we report the results of the computer simulation for clarifying the availability of our proposed method.

We first report the results evaluating the effects of the length of forwarding paths on each proposed algorithm. Next, we report the results evaluating the effects of cache status on each CR.

The parameters used in the evaluations are listed in Table 1. We assumed that WSN consists of fixed sensor nodes employing IEEE802.11 WLAN air interface.

Table 1 Simulation parameters

\begin{tabular}{lc}
\hline Parameter & Value \\
\hline Data rate & $5.5 \mathrm{Mbps}$ \\
SIFS & $10 \mu \mathrm{s}$ \\
DIFS & $50 \mu \mathrm{s}$ \\
Slot & $20 \mu \mathrm{s}$ \\
Contention window size & Min: 31, max: 1,023 \\
MAC header & Data/interest: 24 \\
& ACK: 10 (byte) \\
Frame check sequence & 4 Bytes \\
PLCP header and preamble & $192 \mu \mathrm{s}$ \\
Interest payload & 72 byte \\
Data payload & 250 byte \\
Simulation time & $10 \mathrm{~s}$ \\
Available cache size & 100 \\
Cache algorithm & LRU \\
Arrival process & Poisson arrival \\
$\alpha$ & 0.3 \\
$\beta$ & 0.3 \\
$\mathrm{~N}$ & 20 \\
\hline
\end{tabular}

Figure 5 Simulation topology to evaluate the effects of path length (see online version for colours)

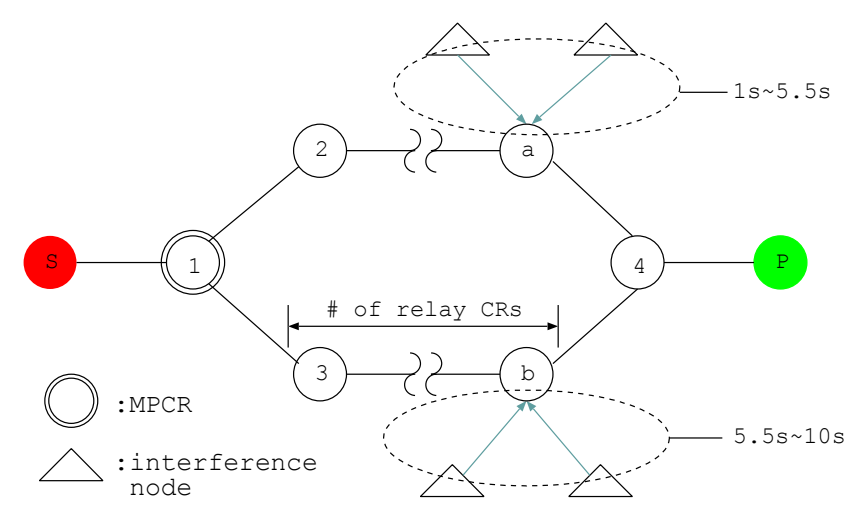

\subsection{Effects of the forwarding path length}

As discussed in the previous section, we proposed the three variants of DCF. Although these variants are proposed for selecting one of the best Interest forwarding paths from its route candidates according to the network conditions, the criterion for the path selection is different among the variants.

Therefore, in this subsection, we evaluate the effects of the forwarding path length on each DCF variant.

Network topologies used for the evaluations are shown in Figure 6. A number of relay CRs among the MPCR (shown as 1) and the interflow CR (shown as 4) are varied according to the evaluation settings.

In addition, for all evaluations, CRs (shown as $\mathrm{a} / \mathrm{b}$ ) before the interflow $\mathrm{CR}$ are located in the range of the interference nodes. In the evaluations, the upper two interference nodes interfere in node a for about 1-5.5 seconds, and the lower two interference nodes interfere in node $\mathrm{b}$ for about $5.5-10$ seconds.

In the evaluations in this section, the data request arrived at node $\mathrm{S}$ at a rate of $100[\mathrm{pkt} / \mathrm{s}]$.

Figure 6 Simulation topology (see online version for colours)

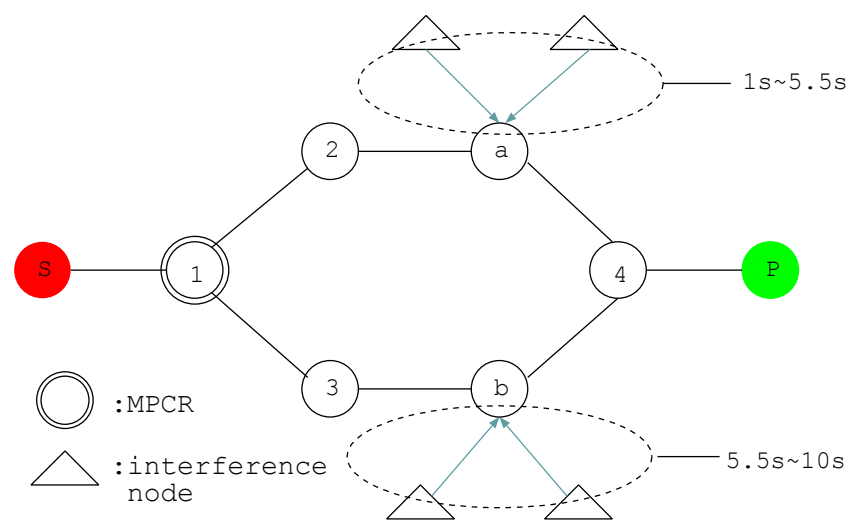

Note: Number of relay CRS $=2$.

Figure 7 Data acquisition rate when the number of relay CRs is two (see online version for colours)

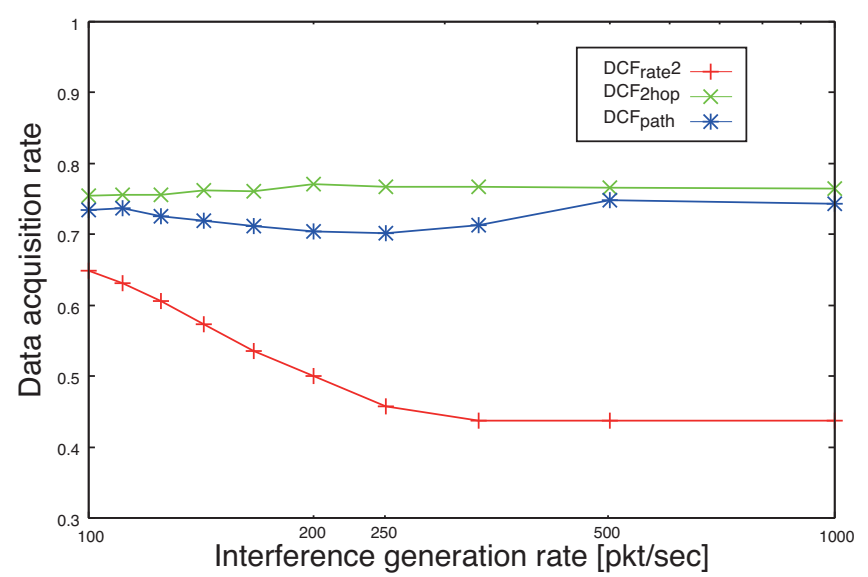

\subsubsection{Characteristics of data acquisition rate (number of relay $C R s=2$ )}

Figure 6 shows the simulation topology where the number of relay CRs is two, and Figure 7 shows the results of data acquisition rate when the number of relay CRs is two. DCF2hop achieves the highest performance than the other two variants because it can investigate the status of a 
wireless link two hops apart from the MPCR, then the MPCR can select the adequate route.

The $\mathrm{DCF}_{\text {path }}$ achieves the second highest performance, and it becomes slightly worse than the $\mathrm{DCF}_{2 \text { hop }}$ because it can investigate the status of a wireless link of CRs belonging to the candidate paths. Then, it can select the adequate path similar to the $\mathrm{DCF}_{2 \text { hop }}$. However, the $\mathrm{DCF}_{\text {path }}$ need a certain period to obtain the status information, whereas the $\mathrm{DCF}_{2 \text { hop }}$ can obtain the status directly from the corresponding node. Therefore, the $\mathrm{DCF}_{\text {path }}$ needs additional time to switch the adequate forwarding path compared with the $\mathrm{DCF}_{2 \text { hop. }}$.

Compared with the two other methods, the $\mathrm{DCF}_{\text {rate2 }}$ indicates a lower data acquisition rate because it cannot obtain the status information of nodes under interferences (nodes $\mathrm{a} / \mathrm{b}$ ). Therefore, the $\mathrm{DCF}_{\text {rate2 }}$ cannot change the adequate forwarding route according to the investigation.

\subsubsection{Characteristics of data acquisition rate (number of relay $C R s=3$ )}

Figure 8 shows the simulation topology where the number of relay CRs is three, and Figure 9 shows the results of computer simulation where the number of relay CRs is three. DCFpath achieves higher performance than the two other methods because it can obtain the status information over the nodes belonging to the path.

Figure 8 Simulation topology (see online version for colours)

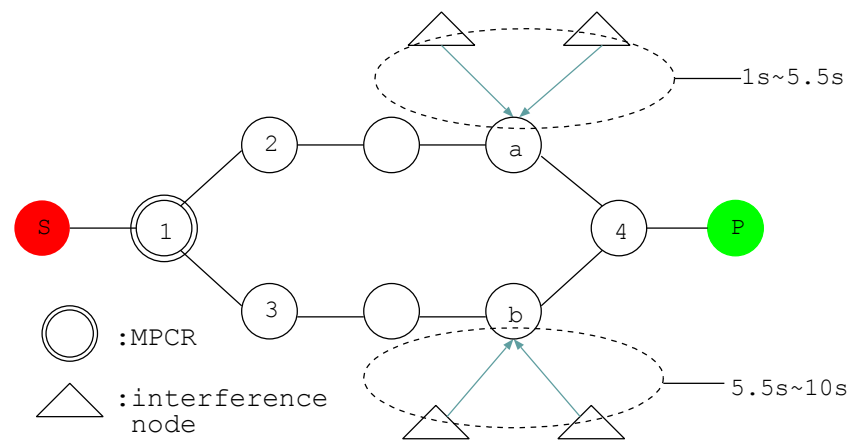

Note: Number of relay CRs $=3$.

Figure 9 Data acquisition rate when the number of relay CRS is three (see online version for colours)

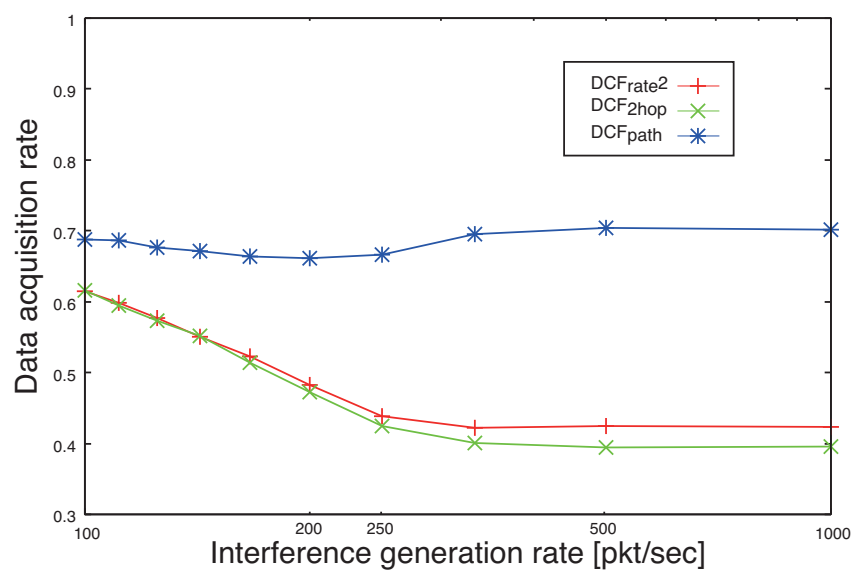

The two other methods, $\mathrm{DCF}_{\text {rate2 }}$ and $\mathrm{DCF}_{2 \mathrm{hop}}$, have lower performance. $\mathrm{DCF}_{2 \text { hop }}$ lost its advantages over the $\mathrm{DCF}_{\text {rate2 }}$ completely because both methods obtain the status information of one or two hops apart from the MPCR node. Then, the status information of nodes $a$ and $b$ cannot be communicated to the MPCR. Hence, both methods cannot select an adequate forwarding route from the two candidate routes.

\subsubsection{Characteristics of data acquisition rate (number of relay $C R s=4$ )}

Figure 10 shows the simulation topology where the number of relay CRs is four, and Figure 11 shows the results of computer simulation where the number of relay CRs is four.

Figure 10 Simulation topology (see online version for colours)

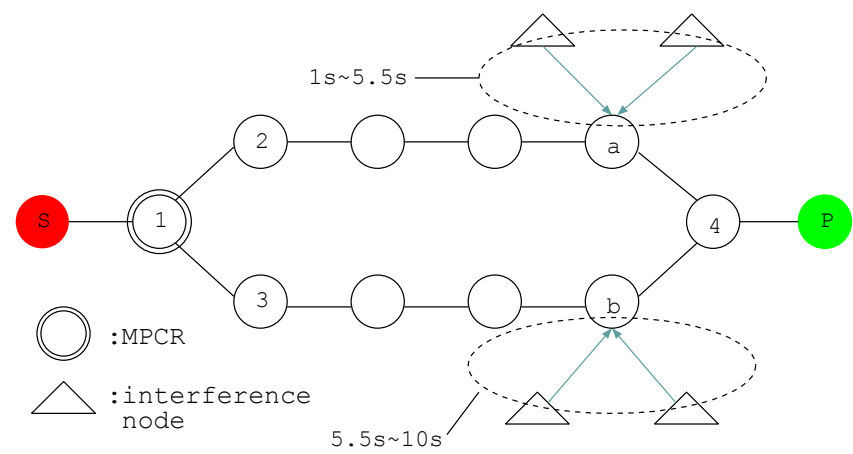

Note: Number of relay $\mathrm{CRs}=4$.

Figure 11 Data acquisition rate when the number of relay CRS is four (see online version for colours)

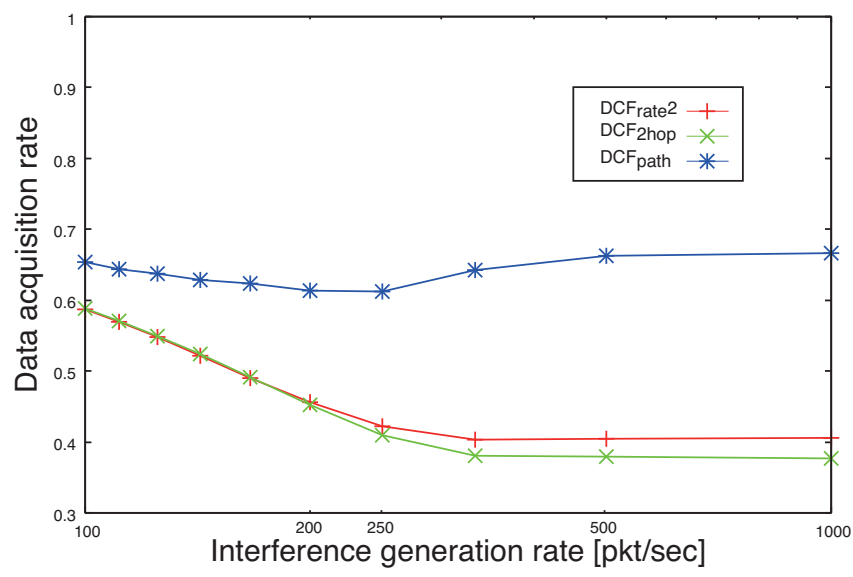

The order of the three methods is almost similar to the case where the number of relay CRs is three. However, the performance of the $\mathrm{DCF}_{\text {path }}$ is slightly lower than the previous evaluation. This phenomenon is caused by need of the $\mathrm{DCF}_{\text {path }}$ to obtain the response from the original server with the specified content. The delay in obtaining the response is increased according to the increase in the number of relay CRs. Therefore, the overhead of the path selection is also increased when the path length is long.

Figure 12 shows the characteristics of data acquisition rate under varying number of relay CRs. The results show 
that the interference transmission ratio of the interfering nodes is fixed at $1,000[\mathrm{pkt} / \mathrm{s}]$.

Figure 12 Data acquisition rate under varying number of relay CRS (see online version for colours)

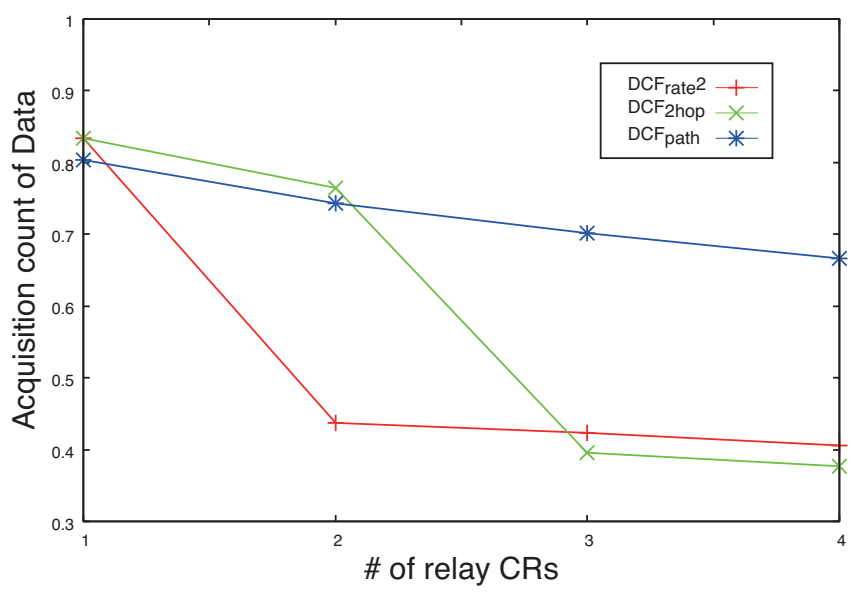

Although the performance is degraded gradually, the DCFpath maintains a better performance compared with the two other methods, where the DCF2hop and DCFrate2 have significantly degraded performance when the number of relay CRs is increased.

Figure 13 Evaluation topology for cache-centric forwarding under string topology (see online version for colours)

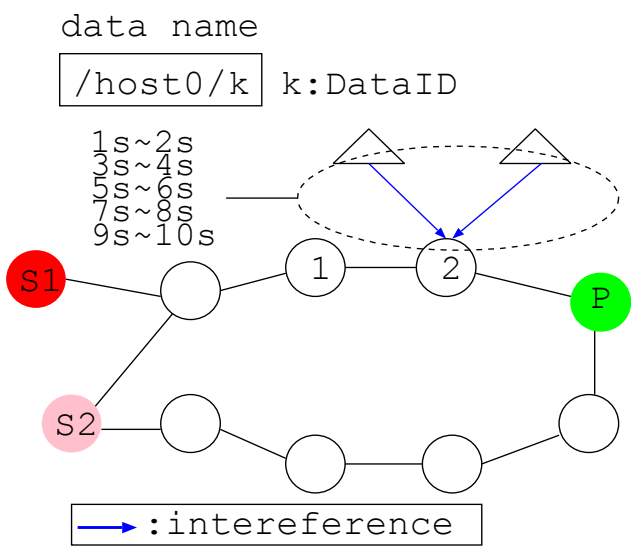

Figure 14 Cache utilisation (see online version for colours)

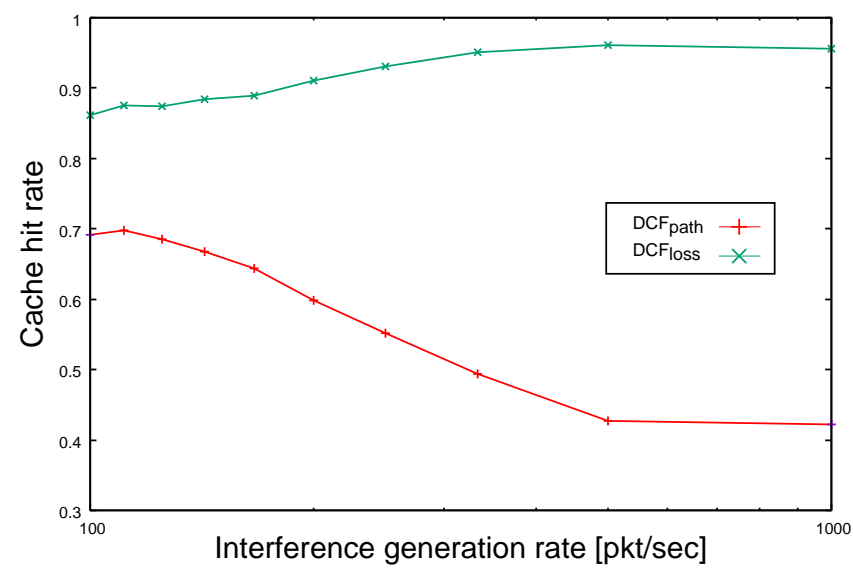

\subsection{Effects of cache-centric forwarding}

\subsubsection{Evaluation of cache-centric forwarding under string topology}

To evaluate the cache-centric forwarding introduced by $\mathrm{DCF}_{\text {loss, }}$ we evaluated the contents by obtaining the performance of the topology shown in Figure 13. Each consumer S1 and S2 requested the interests with the prefix '/host $0 / k$ ' where $k$ is the data ID sequentially increased at each Interest transmission. The two nodes denoted by triangle mark are the interference nodes. These two nodes transmit the interference packet to the relay node denoted by two during the period shown in Figure 13.

The results of the cache hit rate are shown in Figure 14. $\mathrm{DCF}_{\text {loss }}$ achieves higher performance than $\mathrm{DCF}_{\text {path }}$ because it selects the upper route with more useful cached content at the closer relay node with $\mathrm{S} 2$, while the $\mathrm{DCF}_{\text {path }}$ selects the lower route with no useful cache when the $\mathrm{CP}$ exposed the higher packet loss rate in the $\mathrm{DCF}_{\text {path }}$ procedure.

Similarly, the results of RTT shown in Figure 15 confirm that the $\mathrm{DCF}_{\text {loss }}$ achieves smaller RTT by selecting the route with useful cache and smaller hop towards the server.

Figure 15 Round trip time (RTT) (see online version for colours)

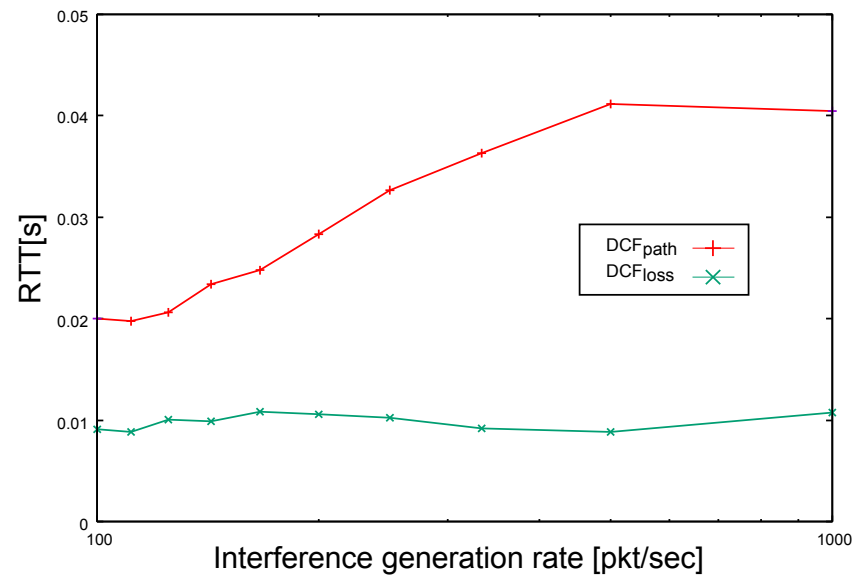

Figure 16 Evaluation topology for cache-centric forwarding under mesh topology (see online version for colours)

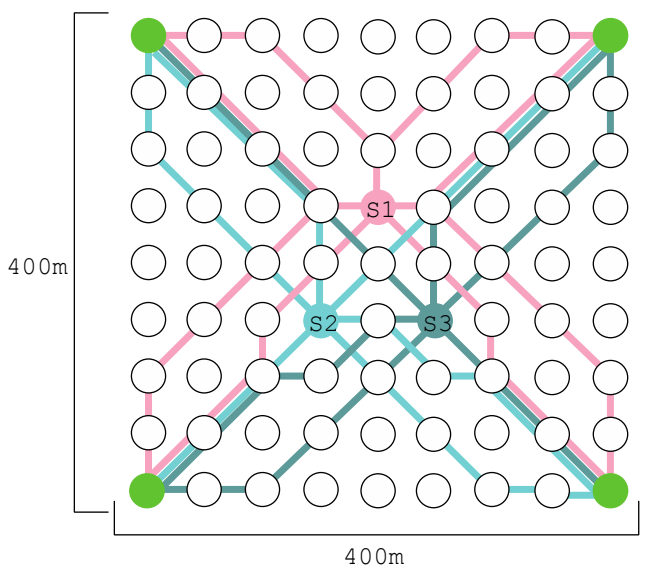




\subsubsection{Evaluation of cache-centric forwarding under mesh topology}

We evaluated the cache-centric forwarding by $\mathrm{DCF}_{\text {loss }}$ under mesh topology. Figure 16 shows the evaluation topology where three consumers are initially given multiple candidate routes. In addition, additional interference nodes were not given. Therefore, only the traffic induced by the Interest/Data exchange generated between the consumers and server will cause the packet collision.

Figures 17 and 18 show the results of cache hit rate and RTT, respectively.

Cache centric forwarding by $\mathrm{DCF}_{\text {loss }}$ achieves higher performance than the $\mathrm{DCF}_{\text {path }}$, which selects the forwarding destination according to the packet loss information only.

Figure 17 Cache hit rate of $\mathrm{DCF}_{\text {path }}$ and $\mathrm{DCF}_{\text {loss }}$ (see online version for colours)

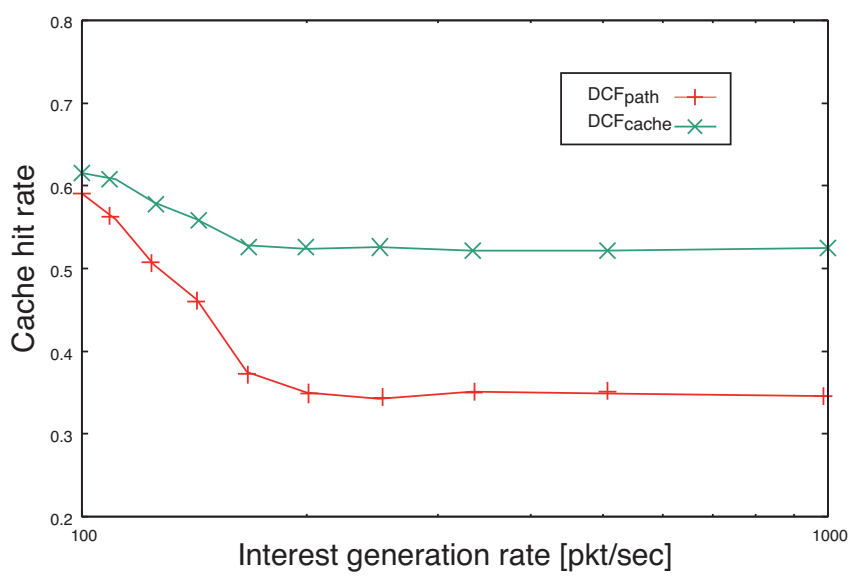

Figure 18 RTT of $\mathrm{DCF}_{\text {path }}$ and $\mathrm{DCF}_{\text {loss }}$ (see online version for colours)

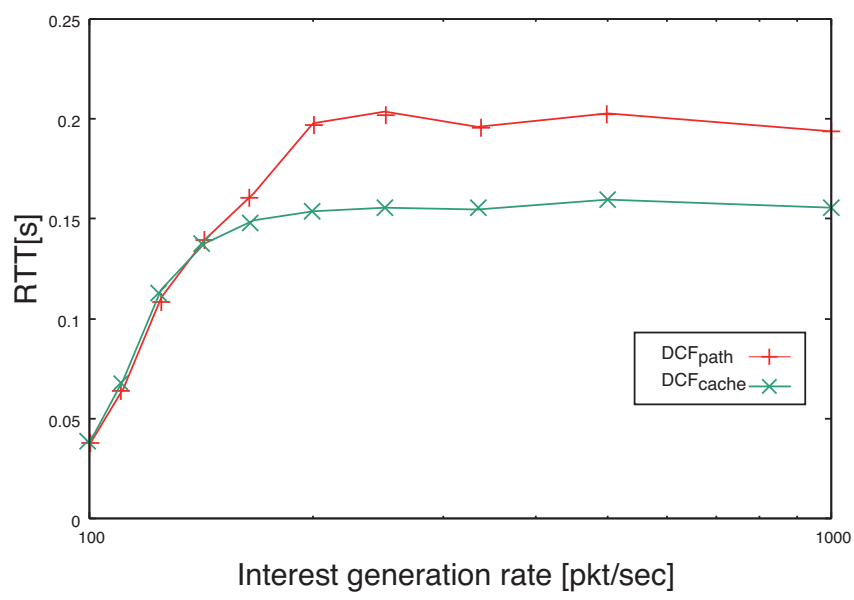

\section{Conclusions}

In this paper, we discussed the implementation of NDN on WSN for coping with the amount of traffic in future content delivery networks induced by IoT technologies. Considering our previous literature, this paper discussed the selection of an adequate forwarding route from candidate routes obtained by the broadcasted Interest. Computer simulation results confirmed that our proposed method called $\mathrm{DCF}_{\text {path }}$, which investigates the adequate route according to the status of all wireless links belonging to the forwarding path, could achieve the most suitable performance.

In addition to investigating the entire wireless conditions of relay nodes, the computer simulation results confirmed that the content-obtaining performance would be improved if the forwarder (MPCR in this paper) selects the route based on the availability of popular contents in the relay nodes.

Finally, because of $\mathrm{DCF}_{\text {loss }}$ proposed in this paper is the cache-centric forwarding control method. Then, this type of data forwarding strategy will effectively improving the cache usage and request/response performance on the next generation broadband networks.

\section{Acknowledgements}

This work was supported by JSPS KAKENHI Grant Number JP17K00132.

\section{References}

Amadeo, M., Campolo, C., Iera, A. and Molinaro, A. (2014) 'Named data networking for IoT: an architectural perspective', Networks and Communications (EuCNC), 2014 European Conference on, Bologna, pp.1-5.

Amadeo, M., Campolo, C., Molinaro, A. and Mitton, N. (2013) Named Data Networking: A Natural Design For Data Collection In Wireless Sensor Networks, Wireless Days (WD), 2013 IFIP, Valencia.

Editors of IEEE (1999) Wireless LAN Medium Access Control (MAC) and Physical Layer (RHY) Specifications, IEEE Std.802.11,.

Hail, M., Amadeo, M., Molinaro, A. and Fischer, S. (2015) 'Caching in named data networking for the wireless internet of things', Recent Advances in Internet of Things (RIoT), 2015 International Conference on, Singapore, pp.1-6.

Intanagonwiwat, C., Govindan, R., Estrin, D., Heidemann, J. and Silva, F. (2003) 'Directed diffusion for wireless sensor networking', IEEE/ACM Transaction on Networking, Vol. 11, No. 1, pp.2-16.

Kuniyasu, T. and Shigeyasu, T. (2017) 'A study on implementation of NDN to WSN', Proceedings of the 31st IEEE International Conference on Advanced Information Networking and Applications (AINA-2017), Taipei, Taiwan, pp.392-398.

Matsui, T., Sekine, H., Hayashi, H., Ohkubo, H., Sunaguchi, H., Matsuo, N., Satoh, Y. (2016) [Invited Talk] Overviews on key technologies to substantialize IoT society, The Institute of Electronics, Information and Communication Engineers Technical Report, Vol. 116, No. 53, pp.77-82.

NDN Project (2010) Named Data Networking (NDN) A Future Internet Architecture [online] https://named-data.net> (accessed 13 July 2016).

Tobe, Y. (2007) 'Trend of technologies in wireless sensor networks', The Institute of Electronics, Information and Communication Engineers Journal B, Vol. J90-B, No. 8, pp.711-719. 
Yamamoto, M. (2014) [Special Invited Talk] Research trends in content oriented networks, The Institute of Electronics, Information and Communication Engineers Technical Report, Vol. 114, No. 18, pp.35-40.

Yu, Y.T., Li, Y., Ma, X., Shang, W., Sanadidi, M.Y. and Gerla, M. (2013) Scalable opportunistic VANET content routing with encounter information, information', Proceedings International Conference on Network Protocols, ICNP.
Zhang, Y., Zhang, H. and Zhang, L. (2014) 'Kite: a mobility support scheme for NDN', 1st International Conference on Information-Centric Networking - (ICN), No. 1, pp.179-180.

Zhang, Z., Ma, H. and Liu, L. (2015) 'Cache-aware named-data forwarding in internet of things', 2015 IEEE Global Communications Conference (GLOBECOM), San Diego, CA, pp.1-6. 